\title{
Actuator management development on ASDEX-Upgrade
}

\author{
O. Kudlacek ${ }^{\mathrm{a}, *}$, W. Treutterer ${ }^{\mathrm{a}}$, F. Janky ${ }^{\mathrm{a}}$, B. Sieglin ${ }^{\mathrm{a}}$, M. Maraschek ${ }^{\mathrm{a}}$, The ASDEX Upgrade team ${ }^{\mathrm{b}}$, The EUROfusion \\ MST1 Team ${ }^{\mathrm{c}}$ \\ a Max Planck Institute of Plasma Physics, Boltzmannstr. 2, 85748 Garching, Germany \\ ${ }^{b}$ see A. Kallenbach et al 2017 Nucl. Fusion 57102015 \\ c see H. Meyer et al 2017 Nucl. Fusion 57102014
}

\begin{abstract}
In future tokamak devices, the control system will have to handle several control goals simultaneously with a limited number of actuators in long and high performance discharges. One of the critical roles of the future control systems will be the management of actuators, which would assign the most convenient available actuators primarily to the control goals of the highest importance at the time. Such a system would consist of a discharge program defining the experiment, a discharge supervisor making automatic high level decisions in real time and a component handling the actuators at a lower level: The virtual actuator, which is a software object responsible for distributing the controller commands to a set of selected actuators. This paper describes the implementation of a virtual actuator for all 8 gyrotrons of ASDEX-Upgrade. We also describe the intended use of the virtual actuator for three experiments: $\beta$ control using ECRH, a disruption avoidance strategy, and electron temperature profile control. The paper also gives an overview of future actuator management developments at ASDEX-Upgrade: extension to all heating sources, inclusion of mirrors for ECRH, and intelligent real time distribution of the actuators between the control goals.
\end{abstract}

Keywords: tokamak, plasma control, actuator management

\section{Introduction}

In the future, large tokamaks will require a control system which will cope with a large number of control goals (control of a physical quantity, e.g. heating power) using a limited number of actuators (a source that is used to achieve the control goal, for example NBI) in a high performance regime. An essential part of such a control system is intelligent actuator management which will be responsible for allocating the most convenient actuators available to the control goals of the highest importance. Besides the activities on ASDEX-Upgrade, the actuator management is being developed for DIII-D [1], TCV [2] and a general theoretical study has been presented in [3].

The first version of the actuator management for ASDEXUpgrade was developed for MHD control and impurity accumulation preemption 4. However, it was restricted to just the $4 \mathrm{ECRH}$ systems with fast mirrors. The control experiments foreseen on ASDEX-Upgrade, such as electron temperature profile $\left(T_{e}\right)$ control [5], current profile control for advanced tokamak discharges, $\beta$ control using various types of actuators or advanced disruption avoidance schemes [6] require a more generic scheme which is applicable to any kind of heating and current drive actuator at ASDEX-Upgrade: 4 ECRH with fast mirrors, $4 \mathrm{ECRH}$ with slow mirrors, $2 \mathrm{ICRH}$ systems, and $8 \mathrm{NBI}$

\footnotetext{
* Corresponding author

Email address: ondrej.kudlacek@ipp.mpg.de (O. Kudlacek )
}

systems. In principle, it should be capable of combining different types of actuators to one control goal. If needed, the scheme should be extendable also to fuelling and impurity control in the divertor region. The scheme of the envisioned control system is shown in Figure 1.

In this scheme, the experimental goals and control priorities are defined in the discharge program. The input from the discharge program will be used to configure the discharge supervisor, an intelligent algorithm which will make high-level decisions in real time by combining the information from the discharge program with the actual state of the plasma and the machine. The discharge supervisor does not necessarily need to run at each time step. The controllers will be responsible for the control algorithms for each controlled quantity and will obtain the necessary measurements directly from the diagnostics and observers and the references either from the discharge program or the discharge supervisor. The controllers will not directly effect a real actuator, but rather a virtual actuator representing a group of real actuators with similar impact. The virtual actuator combines the real actuators that are designated to a controller.

In the AUG Discharge Control System (DCS) [7, the controllers already exist, the discharge supervisor is to be developed later and the virtual actuator has been introduced recently. The functionality of the virtual actuator introduced for the ECRH systems at ASDEX-Upgrade, the interface to the controllers and the first foreseen use in 


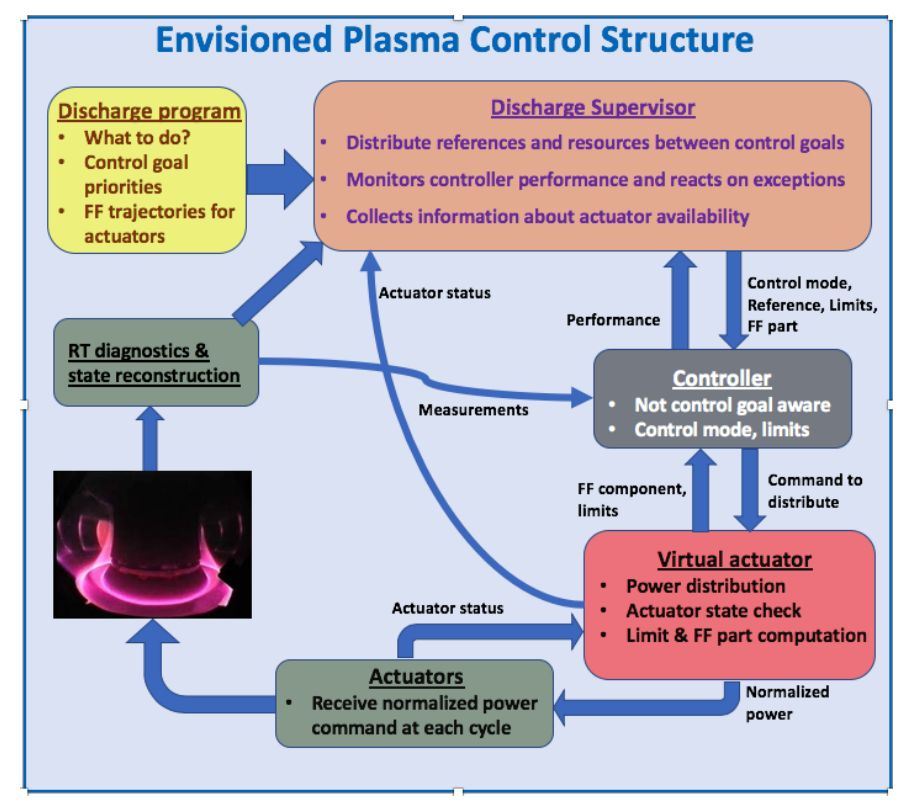

Figure 1: A scheme of the envisioned future tokamak control system and the information flow between individual parts.

experiment are described in this paper.

\section{The virtual actuator}

A virtual actuator is an object in the DCS which takes care of the actuators assigned to particular controller output. In the case of Single Input Single Output controller, one virtual actuator per controller output will be used, in the case of Multiple Input Multiple Output controller, each output has its own virtual actuator. The virtual actuator has been introduced for all 8 ECRH gyrotrons at ASDEXUpgrade. At this stage, the real actuators are assigned to virtual actuators in the discharge program by the person preparing the discharge, who also decides the priority of their use. In the future the discharge supervisor will be able to change the priority or to relocate them to another virtual actuator in real time based on actual needs (see Section 4.3). For example, one could move gyrotrons used for electron temperature control to suppress an NTM.

During discharge program validation, the virtual actuator assures that all real actuators in the virtual actuator exist and each real actuator is strictly just in one virtual actuator. After relocation between virtual actuators will be implemented, this will have to be assured during runtime. If this is not the case, an error is reported and the discharge program is not validated.

The virtual actuator is active in two phases of the real time cycle (see Figure 1). First, it prepares all inputs necessary for the controller, which is run afterwards. After that the controller delivers the command to the virtual actuator. Consequently, the virtual actuator distributes individual commands between the real actuators that are ready to actuate. From now on, we will restrict our de- scriptions to control using ECRH power, for which the virtual actuator has already been introduced.

The DCS controller (see [7] for more information about the DCS controller design) needs to obtain the following inputs from the virtual actuator to run: upper and lower limit for anti-windup and the feedforward command. The virtual actuator first checks the availability of the power sources. For the gyrotrons that are ready to deliver power, the power deposition location check is made: Each of the control channels has a desired power deposition location which is used to configure the virtual actuator. To avoid problems in deposition-sensitive control algorithms, such as electron temperature profile control described in section 3 caused by gyrotrons depositing the power at wrong location (for example due to a mistake in discharge preparation or a different density profile from the expected one), only gyrotrons that point to the right location can be active members of a virtual actuator. The power deposition location is computed for each gyrotron in real time by the beam tracing code TORBEAM [8]. The upper limit for the controller output is the power available in all gyrotrons that are ready to actuate and point to the right location.

Another signal which has to be delivered to the controller is the feedforward (FF) component in the same physical unit the controller is using: Currently, the controllers use Watts, but the number of desired physical units is expected to be extended later (see Section 4.1). The feedforward component is composed from two parts: the FF signal for the virtual actuator and the direct FF command to each individual real actuator. Both can be specified in the discharge program.

The direct commands can be given to any actuators, especially because some diagnostics depend on specific power sources (for example, the charge exchange diagnostic needs power in particular neutral beams to measure properly). Therefore, the power source must be active all the time. The actuators with a pre-specified power waveform can also be members of the virtual actuator, however, the virtual actuator is not allowed to change their power output, though the power delivered by those actuators is included in the power sum delivered by the virtual actuator. The power that has to be delivered due to these direct commands serves as a lower limit for the controller and can not be changed even if the controller required less power.

The other part of the FF component, the FF signal, is traditionally specified in the discharge program in a number of active power sources, not in the physical units used by the controller. The reason is that the maximum power the actuator can deliver changes during the discharge due to the properties of the power sources. In the case that the FF signal would be specified in Watts, the feedforward component would often not be an integer multiple of the number of sources for the whole discharge. This would result in power source modulation, which is unwanted because it increases the risk of the power source trip during the discharge. However, this design choice brings a need of unit transfer from number of sources used by the discharge 
designer to the physical units used by the controller. This action is performed by the virtual actuator as well: The virtual actuator has information about the power deliverable by each of the actuators and it also knows which actuators would deliver power due to the FF component. The unit transfer is performed as a sum of the power in all sources active due to the FF component.

If the number of sources required by the FF signal is higher than the number supplied in the direct commands, the power sources with the highest priority, but without a direct command to them, are used to allocate the desired number of sources. After the required number of sources is allocated, the power that would be provided by them is summed and sent to the controller as the feedforward part.

If the number of sources required by the FF signal is lower than the number of sources in direct commands, the power delivered in the direct commands is considered the FF part of the controller. In the other words, the original FF signal is overwritten by the direct FF commands in such a situation. The direct commands cannot be changed by any action of the controller, only the command on sources allocated due to the FF signal can be changed by a controller action.

After the controller output is computed, the second part of the virtual actuator executes. This part distributes the power commands to the real actuators which are operational and point to the right location. First, the direct commands are passed and the power distributed in them is subtracted from the power request. Afterwards, the remaining power is distributed among the actuators according to their priorities. Each of the real actuators receives a power norm command between 1 (full power) and 0 (no power). If the request to the actuator is different from off/full power and the power source requires only binary on/off command, the power source is modulated: periodically turned on and off (receiving power norm command 0 or 1 ) in such a way that the mean power over a certain period of time corresponds to the required power. The modulation, which used to be performed in the parts of the DCS system responsible for power distribution, is now a distinct function of the virtual actuator.

\section{First practical applications}

Recently, the virtual actuator described in section 2 has been implemented to the DCS system. It has not yet been used in a real experiment, but we have proved its correct functionality in replay mode: a dry-run of the DCS system which allows to replay all real time diagnostics signals as if they were produced in a real discharge and test the behaviour of the control system. After the operation restart, we intend to use the virtual actuator for three applications: $\beta$ control using ECRH, temperature profile control and disruption avoidance.

The $\beta$ control using ECRH is required by I-mode experiments 9 , which require the value of $\beta$ to be kept in a narrow window while avoiding changes in plasma rotation. Due to the latter requirement, the standard ASDEXUpgrade $\beta$ control using NBI is not useful and a single gyrotron is too weak to accomplish this control goal. Therefore, we combine more gyrotrons into a virtual actuator as shown in Figure 2. Obviously, the controller is saturated quickly, since the measurements are not affected by the control action: this example is shown to demonstrate the functionality of the virtual actuator.
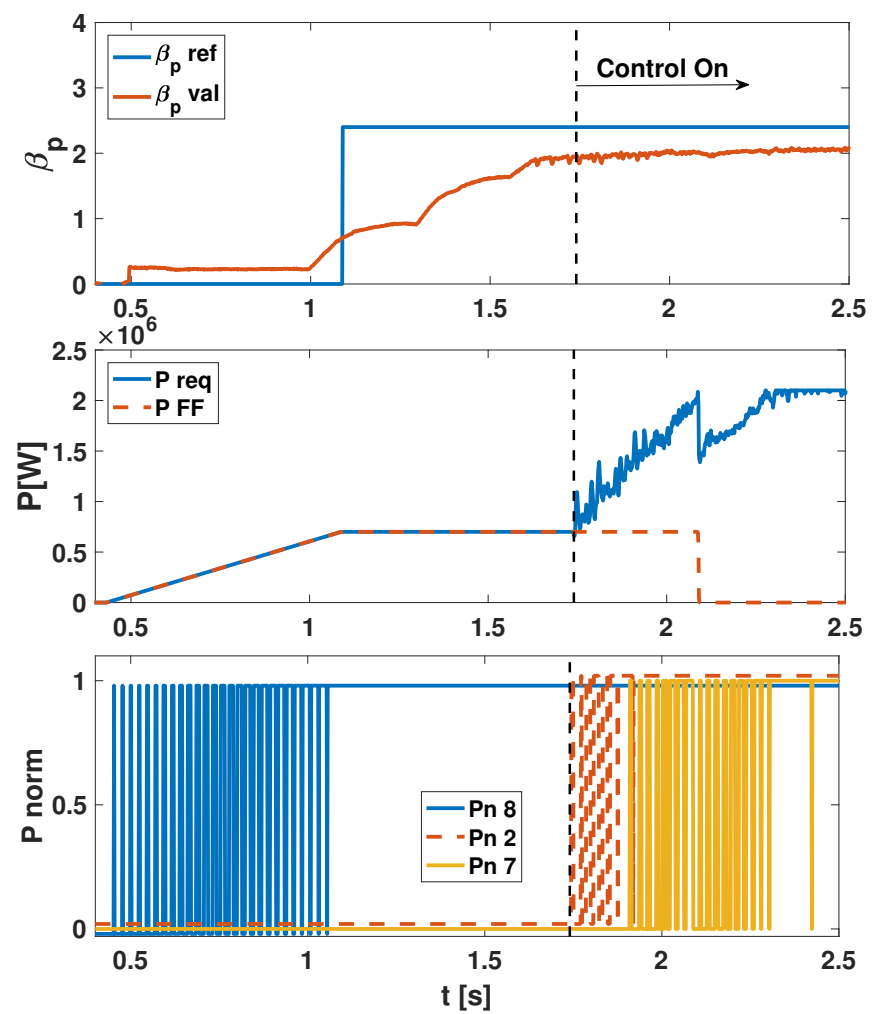

Figure 2: $\beta$ control in DCS replay of discharge 34962 with gyrotrons 8,2 , and 7 assigned to this control goal in the same priority order. The reference and real $\beta$ value are in the top plot. The power required by the controller ( $\mathrm{P}$ req) and the feedforward power requested from the discharge program (P FF) are in the second plot. The third plot contains the power norm (Pn) request on each gyrotron. One can see that the gyrotrons are started exactly in the order of priority and a modulation takes place if full power of the source is not needed.

In Figure 2, one can see that the gyrotrons are started in the priority order as the requested power increases.

There are two further applications where the virtual actuator feature will be used. The first is a disruption avoidance experiment, where the proximity of the plasma state to the pre-defined disruptive region will be monitored 6]. If the plasma state approaches the region, EC power will be applied to recover the discharge. The power will increase with the plasma state coming closer to the disruptive boundary. Originally, this experiment was supposed to be performed just with a single gyrotron. However, the implementation of the virtual actuator enabled the use of a more powerful scheme with several gyrotrons. The dis- 
charge designer will be able to decide on the highest number of gyrotrons that should be used to bring the plasma back to the safe state. If a sufficient number of gyrotrons is assigned to the virtual actuator for this control goal, possibly tripped gyrotrons can be replaced by those with lower priority. This replacement would reduce the impact of such a failure on the experiment.

Virtual actuators will be also used for electron temperature profile control. In this experiment, the $T_{e}$ profile is kept constant during the discharge actuating the EC power deposited at two different radial locations selected before the discharge. In the first experiments reported in [5], only one gyrotron per channel was used. Due to the lack of actuating power, the controller was in saturation about $50 \%$ of the time it was active. Naturally, this has significantly decreased overall performance of the controller. In future experiments with the $T_{e}$ profile control, each of the control channels in this experiment will have its own virtual actuator with gyrotrons selected by the discharge designer. Together with convenient FF waveforms applied to the virtual actuators, the time the controller is in saturation will be reduced.

\section{Outlook}

As indicated in section 1 , implementation of the virtual actuator to the DCS system was just the first step of a long-term development. We intend to gradually increase the flexibility of the control system. The following steps, not necessarily in the order presented in this paper, are expected in the following years:

\subsection{Increase the number of quantities treated by the vir- tual actuator}

Currently, the virtual actuator is suited only for experiments with heating power control. However, in the future we will also need to control other quantities, such as the driven current for q-profile control or momentum input for plasma rotation control. We intend to develop the control system in such a way that the virtual actuator can be used to compute the controller limits, the FF command for the controller and to distribute the controller command between individual actuators for more quantities of interest.

\subsection{Extension to ECRH mirrors}

Presently, only the gyrotron power is controlled by the virtual actuator. The power deposition location is determined by the ECRH mirror settings and can not be changed by the virtual actuator, though this feature would be required by several control goals such as NTM control or current profile control using mirror movement. In the future, we intend to point all mirrors corresponding to the member gyrotrons of the virtual actuator to the desired power deposition location.

\subsection{Enable relocation of real actuator}

In current version, the virtual actuator has a fixed set of real actuators specified in the discharge program. However, as the power required by the control goals changes over time, it would be more efficient in terms of power source use to move the gyrotrons between virtual actuators. This will already require a discharge supervisor responsible for the movements.

\subsection{Extension to $N B I$ and $I C R H$}

We plan to use the virtual actuator to command individual NBI and ICRH sources and to enable the combination of more types of power sources in a single virtual actuator. The latter will require a discharge supervisor which would guarantee that each virtual actuator will contain just real actuators suitable for the control goal (for example no NBI for the NTM control). A direct benefit of this is, for example, in the impurity accumulation avoidance experiments. Normally, the plasma centre is heated by ECRH to avoid impurity accumulation, but this method becomes useless at high densities when the EC wave cannot access the plasma centre any more. In such a situation, the missing EC power can be replaced by IC heating.

\subsection{Human friendly interface development}

As we intend to use the actuator management as a routine tool, it is crucial to prepare a user friendly human interface, which will become more important with the increased complexity of the tool. The configuration has to be transparent even to a person without deep knowledge of its internal structure. We foresee the possibility of rapid prototyping using the flight simulator Fenix currently in development for ASDEX-Upgrade [10, [1].

\section{Acknowledgements}

This work has been carried out within the framework of the EUROfusion Consortium and has received funding from the Euratom research and training programme 20142018 under grant agreement No 633053. The views and opinions expressed herein do not necessarily reflect those of the European Commission.

\section{References}

[1] D.A. Humphreys et al. Integrated plasma control in DIII-D, Fusion Sci. Technol., 48 (2) (2005), pp. 1249-1263

[2] T. Vu et al, Tokamak-agnostic actuator management for multitask integrated control with application to TCV, in preparation for Nuclear Fusion

[3] E. Maljaars, F. Felici, Actuator allocation for integrated control in tokamaks: architectural design and a mixed-integer programming algorithm, Fusion Eng. and Des., 122 (2017), pp. 94-112

[4] C.J. Rapson et al., Actuator management for ECRH at ASDEX Upgrade, Fusion Eng. Des., 96 (2015), pp. 694-697

[5] F. Felici et al, Model-based design, simulation and testing of an electron temperature profile controller on ASDEX-Upgrade, submitted to Nuclear Fusion

[6] M Maraschek et al 2018 Plasma Phys. Control. Fusion 60014047 
[7] W. Treutterer et al, ASDEX Upgrade's new plasma control scheme, Fusion Engineering and Design 81 (2006) 1927-1931

[8] E. Poli et al. , Comput. Phys. Commun. 225, 36 (2018)

[9] T. Happel at al, The I-mode confinement regime at ASDEX Upgrade: global properties and characterization of strongly intermittent density fluctuations, Plasma Phys. Control. Fusion 59 014004

[10] F. Janky et al, AUG flight simulator development, P4.030, this conference

[11] W. Treutterer et al, Concepts of the new ASDEX-Upgrade flight simulator, P2.038, this conference 